\title{
Sequence alignment of extra chromosomal deoxyribosenucleic acid base pairs of bacteria and plant: possible mechanism of genome rearrangements
}

\author{
Amruta Samal and Gopala Rao T.V.* \\ *Department of Biotechnology, G.I.E.T., Gunupur - 765022, Orissa, gopalraotv@yahoo.com
}

\begin{abstract}
Computer generated sequence alignment became a conventional tool in bioinformatics. SIM is one of the methods for determining similarity between two sequences through a pair-wise sequence alignment. However, most of the conventional tools lack the ability of incorporating biological properties into the alignment result which often leads to incorrect alignment. In this paper, we used a pair-wise alignment system called SIM that incorporates biological properties into the algorithm so that it can suggest an appropriate sequence alignment based on the given nucleotide base pairs. SIM indicated nucleotide sequences alignments and suggested massive rearrangements of extra chromosomal, which can be extrapolated to total genome rearrangements.
\end{abstract}

Key words- sequence alignment, plastid, plasmid, genome, base pair rearrangements

\section{Introduction}

There are reports that maximum of medicinal plant extracts are containing of protein or peptide antibiotics [1, 2]. They inhibit the growth of micro organisms [3]. The typical plasmids are of Escherichia coli and Bacillus species, are matching or overlapping from the range of minimum of 4 to 15 base pairs of the plant plastid. This alignment of plasmids of Bacillus sps. and E. coli with and without gap frequency was observed for resistance and sensitivity of anti bacterial with crude extracts of plant leaf [Tables -1 and 2]. Here, in our observation we compared two microbes' plasmid genome sequences for alignment with plastid sequence of Alstonia scholaris, where all are extra chromosomal. However, the plant leaf of crude extract of Alstonia scholaris is inhibiting the growth of E. coli and Bacillus sps., [un published data of a project dissertation]. Bacterial plasmids are containing antibiotic resistance genes and the best example for that is pBR322 of E.coli [4]. Several investigations are there for the synergistic effects between physiochemical and antibiotics [5].

\section{Results and conclusions}

The evaluation of the biological activity of plant extract is on standard micro organisms as well as multi-drug resistant bacteria isolated from hospitals [5]. Thus, the sequence alignment by using SIM tool [SIM is a program which finds a user-defined number of best non-intersecting alignments between two nucleotide sequences or within a sequence. SIM finds $k$ best nonintersecting alignments between two sequences or within a sequence using dynamic programming techniques. The alignments are reported in order of decreasing similarity score and share no aligned pairs. SIM requires space proportional to the sum of the input sequence lengths and the output alignment lengths], may indicate that the nucleotide base pairs of the plant plastid align automatically by rearranging as for the requirement, and for the protection against bacteria and fungi. If plants are producing peptide antibiotics for their protection, substantially the same extracts are being able to control the microbial infections of animals including human. The probable mechanism is observed in matching of Bacillus spes., plasmid genes with plant $A$. scholaris plastid genes, at the level of non intersecting base pairs. The two examples of genes have been taken here for alignments are of ribosomal protein and an enzyme ribulose 1, 5 diphosphatase. The comparison of which we have taken sequence alignment base pairs of plasmids of Bacillus spes and $E$.coli with base pairs of ribosome protein genes and one of the enzymes 1,5 ribulose diphosphatase of plastid of plant $A$. scholaris, which are initially required for nucleic acids synthesis and for the synthesis of enzymes for sequence arrangements show matching similarities without gap frequency [Tables-1 and 2]. In the first comparison the identity of 30 residues overlapping was around $70 \%$ without gap frequency [Table -1]. This non intersecting alignment may be further concluded for proving that there are heavy rearrangements of the nucleic acid base pairs, similarities and dissimilarities match during replication, transcription and translation before the splicing and which was for the better expression within fractions of nano seconds. Randomly if we compare alignment of deoxyribonucleic acid sequences and proteins, we can conclude massive rearrangements for the protection and cell division of cells [6 - 8]. These massive rearrangements can be answering to the lot of wet laboratories results of siRNA and oligodeoxynucleotides (ODNs) in interference of the sequences after intersecting changes of the genomes [9]. One of the examples of genome rearrangements of a ciliate Tetrahymina, that there are extensive and reproducible genome rearrangements of the germ line during conjugation [10]. Intersecting and alignments may be further evident for the rearrangements of nucleotides sequences. It may be randomly taking place in the cell. That's how the rearrangements of splicing taking place for the post transcriptional modifications. Another example of SIM4 [sample images from multiple alignment] which will solve the problem of efficiently aligning a transcribed and spliced deoxyribonucleic acid sequence [mRNA] with a 
genome sequence containing that gene, allowing for introns in the genome sequence [taking into account consensus splice signals] and a relatively small number of sequence errors [11]. Since SIM is for multiple alignments when compared to the BLAST, SIM toll was preferred in this paper. Either it may be post transcriptional modifications or post translational modifications for the expression of different proteins in the cell, the rearrangements of nucleotides are like a wave and are possible in the cell's nucleic acids. The cells and organelles must be having all sorts of transitory enzymes for the sake of rearrangements. With this one can conclude that the two genes that of ribose protein and 1,5- ribulose diphosphatase are proved as typical examples which may be for the inter species comparison of deoxyribonucleic acid sequence alignment of Bacillus spes, E.coli and $A$. scholaris. Further, it can be concluded that by comparing genome sequence alignments of different genera and species which may be evident for massive rearrangements of deoxyribonucleic acid as inter-specially before or after DNA integrated into the host genome. Using the parameters for Table 1A, 1B, 2A, 2B. Number of alignments computed: 20 , Cost of a matching aligned pair: 1., Cost of a transition: -1., Cost of a transversion: -1. ., Gap open penalty: 6.0, Gap extension penalty: 0.2 . The sequence alignment of minimum and maximum number of base pairs using SIM parameters.

Table 1A- The table shows identity of residues maximum of 29 and minimum of 10 . The random pick up of sequence alignments of 2 different percentage of identity (one with $72.4 \%$ and second with 100\%) in search of Bacillus plasmid base pairs with that of plastid of Alstonia scholaris ribosomal protein gene base pairs.

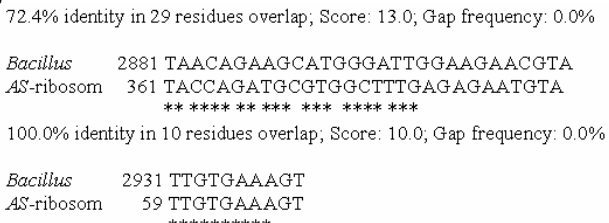

Table 1B- The table shows $69.7 \%$ identity in 33 residues and $100 \%$ identity in 11 residues of E.coli plasmid versus Alstonia scholaris (AS) ribosomal gene with above said parameters of SIM.

$69.7 \%$ identity in 33 residues averlap; Score: 10.2; Gap frequency: $12.1 \%$

E.coli 2849 AAAATCAACAA---CTTAAAAAAGGGGGGTAC AS-ribosom 331 AATATCAGCAAAGCCCTTCACAAATGTGGGTAC $100.0 \%$ identity in 11 residues overlap; Score: 11.0 ; Gap frequency: $0.0 \%$

E.coli 1482 GAAAGTGAGGG

AS-ribosom 63 GAAAGTGAGGG

The sequence alignment of minimum and maximum number of base pairs using SIM tool.
Table $2 A$ - shows identity of residues maximum of 35 and minimum of 11 . The random pick up of sequence alignments of 2 different percentage of identity (one with $80 \%$ and second with 100\%) in search of Bacillus plasmid base pairs with that of Alstonia scholaris (AS) plastid protein gene base pairs. $80.0 \%$ identity in 35 residues overlap; Score: 15.8, Gap frequency: $2.9 \%$

Bacillus 2851 AAATTTTATTGGGAAACATATGCGAAATCATAACA AS-ribulos 24 AAATTTTGTTGCGAAAATTAT-CGAATTCAAAACA

$100.0 \%$ identity in 11 residues overlap; Score: 11.0 ; Gap frequency: $0.0 \%$

Bacillus 162 CCTTTTTTTTT

AS-ribulos 374 CCTTTTTTTTT

Table $2 B$ - The table shows $65.7 \%$ identity in 35 residues and $100 \%$ identity in 11 residues of E.coli plasmid versus plastid of Alstonia scholaris (AS) ribulose 1,5-diphosphatase gene with above said parameters of SIM.

$65.7 \%$ identity in 35 residues overlap; Score: 11.0; Gap frequency: $0.0 \%$

E.coli 2580 TGTGCAATGTAACATCAGAGATTTTGAGACACAAC AS-ribulos 413 TGTACATTTCCACATCTAGGATTTACATATACAAC

100.0\% identity in 11 residues overlap; Score: 11.0 ; Gap frequency: 0.0\%

E.coli 3715 TTGGGTTGCGC

AS-ribulos 519 TTGGGTTGCGC

\section{Acknowledgement}

The authors are very much thankful to the management of G.I.E.T., Gunupur, Orissa, India for providing facilities.

\section{References}

[1] Janakat S., Al Fakhiri S. and Sallal A.K. (2004) Phytother Res. 18(10).

[2] Janakat S.M.,Al-Kakhiri S.M. and Sallal A.K. (2005) Saudi Med J. 26(6), 952955.

[3] Sabir M.S., Ahmad D.S., Hussain I.M.and Tahir K.M. (2007) Saudi Med.J. 28 (2), 259-263.

[4] Chakraborthy G. (1998) In Book Lab Mannual, 54-56

[5] Arias M.E., Gomez J.D., Cudmani N.M., Vottuoni M.A. and Isla M.I. (2004) Life Sci., 75 (2) 191-202.

[6] TangY., Schon E.A., Wilichowsk E., Vazquez M.E., Davidson E. And King M.P. (2000) Mol.Biol.Cell. 11 (4), 1471-1485.

[7] Shao R., Dowton M., Murrell A. and Barjer S.C. (2003) Mol.Biol.Evol. 20 (10), 1612-1618.

[8] Sylvie C., Kerry A.L., Arun K.A. and Maliga P. (2003) Plant J. 35(6), 753762.

[9] Bass B.L. (2001) Nature 411, 428-429.

[10] Duharcourt S. and Yao M.C. (2002) Eukaryotic Cell (2), 293-303.

[11] Florea L., Hartzell G., Zhang Z., Rubin G.M. and Miller W. (1998) Genome Res. 8, 967-974. 